testing we suggest that patients should receive different letters, according to exposure, explaining why testing should be considered. Patients who have undergone highly exposure prone invasive procedures-by analogy with hepatitis B virus transmission-should be recommended to undergo HIV testing. Patients whose risks of exposure are slight should also be offered testing - accumulated data from many such patients would provide a better basis for reassurance. Notification letters should contain a special HIV surveillance test request form and the Association of British Insurers waiver concerning negative HIV tests in these circumstances, which has proved a source of confusion during previous exercises. ${ }^{5}$ The proportion of notified patients who underwent testing should be registered and results analysed according to date and type of operative procedure undertaken: the risk of HIV transmission may depend on the stage of the health care worker's infection.

The new guidelines envisage a series of time consuming, costly, and emotionally charged notifications undertaken with the justifiable aim of reassuring patients at a low but currently unquantified risk. Far better that in the course of such laborious exercises, useful data emerge which can provide patients with genuine reassurance and can assist the develop- ment of safer forms of surgery - to the benefit of surgeon and patient alike.

A GRAHAM BIRD

Department of Immunology,

Churchill Hospital,

Oxford OX3 7LJ

Consultant immunologist

MRC Biostatistics Unit,

SHEILA M GORE

Institute of Public Health,

Cambridge CB2 2SR

1 Department of Health. AIDS-HIV infected health care workers: guidance on the management of infected health care workers (interim). London: Department of Health, 1993.

2 UK Health Departments. AIDS-HIV infected health care workers: practical guidance on notifying patients. London: UK Health Departments, 1993.

3 Tonks A. Revised guidelines for HIV infected health workers. BMf 1993;306:1023.

4 Working Group of the Royal College of Pathologists. HIV infection: hazards of transmission to patients and health care workers during invasive procedures. London: Royal College of Pathologists, 1992.

(a) during surgery. BMf 1991;303:351-5.

6 Heptonstall J. Outbreaks of hepatitis B virus infection associated with infected surgical staff. Communicable Disease Repon 1991;1:R81-5.

Communicable Disease Report 1991;1:R81-5.
Tokars JI, Bell DM, Culver DH, Marcus I, Mendelson MH, Sloan EP, et al. Percutaneous injuries Tokars JI, Bell DM, Culver DH, Marcus I, Mendelson
during surgical procedures. $¥ A M A$ 1992;267:2899-904.

8 MMWR Update: investigations of patients who have been treated by HIV infected health care workers. MMWR 1992;41:344

9 Liesielski C, Marianos D, Ou CY, Dumbaugh R, Witte R, Berkelman R, et al. Transmission of human immunodeficiency virus in a dental practice. Ann Int Med 1992;116:798-805.

\title{
Improving the management of superficial bladder cancer
}

\author{
Fewer routine check cystoscopies?
}

Two thirds of bladder tumours present as superficial disease, in which the tumour either is confined to the bladder mucosa (stage pTa) or invades only into the submucosa, sparing the bladder muscle (stage pT1). Once the initial tumour has been diagnosed and treated, further surveillance is considered necessary because of the risk of recurrence, which occurs in about three quarters of patients, ${ }^{1}$ and invasion of muscle, which occurs in up to a tenth of patients. ${ }^{2}$ Hence the "check cystoscopy," which is such a large part of the work of most urology departments.

Traditionally, this has been performed every three months after diagnosis, the interval increasing after a year of freedom from recurrence, but cystoscopies continue at least annually for several years, if not for life. Until recently, in Britain these examinations were performed mainly under general anaesthesia, usually as a day case procedure. Elderly and unfit patients (a substantial proportion) often needed admission.

This seems good preventive medicine, but urologists are now reconsidering their standard practice. ${ }^{3}$ Any benefits from regular cystoscopy are achieved at a price, both to the patient and to the NHS. Many patients are disturbed by the whole process, and some experience considerable morbidity after a cystoscopy. Is regular surveillance effective? Most check cystoscopies show no abnormality. In a recent study in which the average follow up consisted of eight cystoscopies over about six years the median number of cystoscopies showing an abnormality was two. ${ }^{4}$ A negative result, although reassuring, represents an unnecessary procedure. Perhaps the ultimate treatment failure in superficial bladder cancer occurs when a patient whose tumour never recurs is subjected to nine or ten cystoscopies in the five years after initial treatment.

In preventing the development of tumour invading into muscle regular cystoscopy is unhelpful, ${ }^{3}$ not least because 80 $90 \%$ of such tumours occur in patients without pre-existing superficial disease. Truly superficial, stage pTa, tumours rarely progress, and since endoscopic management consists of treating recurrences after they have occurred there is little evidence that delaying their diagnosis is harmful. The assessment and complete resection of the presenting tumour remains critical and must be done by someone with urological experience. Extensive or multifocal tumour may create special problems. Unsuspected muscle invasion (pT2) and poorly differentiated pT1 tumours must be identified. How then should the remaining patients be followed up?

Ideally, it is better to prevent rather than treat recurrent disease. In a Medical Research Council trial intravesical instillation of mitomycin after diagnosis and treatment of the presenting tumour reduced the risk of subsequent recurrence, especially if it was repeated at the first four three monthly check cystoscopies. ${ }^{5}$ Routine prophylactic chemotherapy has not, however, become common practice in Britain. Increased use of flexible cystoscopy under local anaesthesia will reduce the cost and inconvenience of each examination. ${ }^{6}$

Of most importance is a re-evaluation of check cystoscopy programmes. Are patients undergoing cystoscopy too frequently? Do all patients require the same follow up regimen? Although the most potent harbinger of deep invasion is a pT1 tumour, ${ }^{7}$ various features of the presenting tumour have prognostic importance for recurrence. ${ }^{8}$ In a review of data from the Medical Research Council's trials the two most important indicators were the presence of single or multiple tumours at diagnosis and the presence of tumour at the first three month follow up cystoscopy. ${ }^{9}$

On this basis, patients were divided into three prognostic groups, with a different follow up regimen recommended for each. In particular, in those with a single tumour at presentation and no abnormality on three month cystoscopy annual follow up cystoscopy (perhaps with a flexible cystoscope) was considered to be sufficient. A selective approach would allow resources to be concentrated on those most at risk. These in turn are the patients for whom measures such as intravesical chemotherapy should be considered. In time, establishing a formula that will calculate the optimum follow up interval for each patient may be possible. ${ }^{4}$ 
This will not put urologists out of work; patients with poorly controlled disease will still tax their skills. Identifying and preventing tumour progression remains a problem. With a reduction in the time spent on unnecessary cystoscopies the management of superficial bladder cancer will be better for patients and more rewarding for urologists.

One final point. In private medicine a patient with a bladder tumour is considered a good investment who will provide a steady income for several years. Similarly, urological provider units will now gain much of their income from managing superficial bladder tumours. In future, purchasers of health care may well express an interest in how this is done.

DAVID KIRK Consultant urologist
1 Greene LF, Hanash KA, Farrow GM. Benign papilloma or papillary carcinoma of the bladder? f Urol 1973;110:205-7.

Cutler SJ, Heney NM, Freidell GH. Longitudinal study of patients with bladder cancer: factors associated with disease recurrence and progression. In: Bonney WW, Prout GR, eds. Bladder cancer. Baltimore: Williams and Wilkins, 1982:35-46. (AUA Monograph Vol 1).

3 Abel PD. Follow-up of patients with "superficial" transitional cell carcinoma of the bladder: the case for a change in policy. $B r f$ Urol (in press).

4 Gulliford MC, Burney PGJ, Petruckevitch A. Can efficiency of follow-up of superficial bladder cancer be increased? Ann R Coll Surg 1993;75:57-61.

5 Tolley DA, Hargreave TB, Smith PH, Williams JL, Grigor KM, Parmar MKB, et al. Effect of intravesical mitomycin $\mathrm{C}$ on recurrence of newly diagnosed superficial bladder cancer. $B M \mathcal{F}$ 1988;296:1759-61.

6 Fowler CG, Badenoch DF, Thakar DR. Practical experience with flexible cystoscopy in outpatients. Brf Urol 1984;56:618-21.

7 Abel PD. Prognostic indices for superficial bladder cancers. In: Waxman J, Williams G, eds. Urological oncology. London: Edward Arnold, 1992:154-66.

8 Heney NM, Nocks BN, Daly JJ, Prout GR Jr, Newall JB, Griffin PP, et al. Ta and T1 bladder cancer: location, recurrence and progression. Br f Urol 1982;52:152-7.

9 Parmar MKB, Freeman LS, Hargreave TB, Tolley DA. Prognostic factors for recurrence and followup policies in the treatment of superficial bladder cancer: report from the British Medical Research Council subgroup on superficial bladder cancer (Urological Cancer Working Party). f Urol 1989;142:284-8.

\title{
Screening for ovarian cancer
}

\author{
Still a long way to go
}

Because ovarian cancer is usually asymptomatic until widely disseminated it is commonly diagnosed at an advanced stage and is frequently lethal. Most early stage ovarian cancers are detected incidentally on routine pelvic examinations, and five year survival rates of over $90 \%$ can be achieved for localised disease. This fact, together with the minimal improvements achieved in the past 20 years in mortality with aggressive treatment of advanced disease, has awakened interest in the early detection of ovarian cancer by screening.

Ovarian cancer has a low incidence ( 15 per 100000$)$, which raises questions about the usefulness of screening in the general population (even though ovarian cancer is responsible for most deaths from genital tract malignancies in developed countries). To justify a screening programme, the disease needs to be reasonably common, and screening methods with a high degree of specificity and sensitivity need to be available. It also helps if there is an identifiable precursor of the invasive disease. Subsets of patients have been identified who are at a higher risk for developing ovarian cancer, and screening these patients may prove more cost effective.

Women with a family history of ovarian cancer are one such group. Studies suggest that women with one first degree relative with the disease have a 5\% risk of developing ovarian cancer'; with two affected close relatives they have about a $30 \%$ lifetime risk. ${ }^{2}$ Screening may therefore benefit these women but, even if screening was effective, its impact on the disease as a whole would be minimal, as familial cancers make up less than $3 \%$ of all ovarian cancers.

The only two screening tests for ovarian cancer that have been studied in any detail are pelvic ultrasonography and serum CA 125 concentrations. The two papers published in this week's journal use these techniques differently (p 1030, p 1025 ). The paper by Jacobs and colleagues deals with routine screening by measuring CA 125 concentrations in 22000 apparently healthy postmenopausal women. Those who had raised concentrations were then studied using real time transabdominal ultrasonography, and if this was abnormal, a laparotomy was performed. ${ }^{3}$ Of this large group who were screened, only 11 patients had raised CA 125 concentrations, an abnormal scan, and ovarian cancer at laparotomy. Of the 11 ovarian cancers, seven were stage III or IV. Seven patients with a normal serum CA 125 concentration subsequently developed ovarian cancer, two within 12 months of screening. Thus the protocol detected only four potentially curable cancers (the ultimate reason for screening), detected seven incurable tumours, and failed to detect another seven. At a conservative cost of $\$ 200$ per scan, the four potentially curable cancers were diagnosed at a cost exceeding $\$ 1 \mathrm{~m}$ a case.

The study by Bourne and colleagues used colour flow imaging with transvaginal ultrasonography targeted to patients with a family history of ovarian cancer. ${ }^{4}$ Of 1601 patients screened, $61(3.8 \%)$ had a positive result and had laparotomy or laparoscopy. In this group six ovarian cancers were diagnosed, five being stage I(a). Three of the five stage I patients had "borderline" tumours, which may have been diagnosed by subsequent clinical examination before spread occurred. In the intervals between screening, five ovarian or peritoneal cancers developed. The probability of detecting ovarian cancer using this technique was estimated to be 1 in 10 , improving to 1 in 6 if only complex persistent cysts were considered to be potentially malignant.

These two new studies suggest that we have a long way to go before effective screening for ovarian cancer is established. We have first to develop tumour markers or panels of markers with more specificity than CA 125 . It is estimated that CA 125 concentration is $>65 \mathrm{U} / \mathrm{ml}$ in only half of stage I and II ovarian cancers. ${ }^{5}$ Numerous benign conditions may cause false positive results and the positive predictive value of the test on its own is low. In a study of $\mathbf{5 5 0 0}$ patients screened with annual estimations of CA 125 concentration only six ovarian cancers were found out of 175 with raised concentrations and three cancers developed in the group with normal serum CA $125 .^{6}$

Screening using transvaginal ultrasonography seems to lower the rate of false positive scans when compared with abdominal ultrasonography ${ }^{5}$ but it is still not accurate enough as a screening technique. Because of the increased angioneogenesis of malignant tumours colour flow Doppler sonography has been used to distinguish between benign and malignant tumours, and a "morphology index,"-based on tumour volume, cyst wall structure, and structure of the septa-may increase the specificity of transvaginal ultrasonography as a screening method ${ }^{8}$. When this method was used to analyse scans on 3220 asymptomatic postmenopausal women, however, 44 patients had a laparotomy for a persisting abnormality, and only three had ovarian cancer, one of which was already stage III. ${ }^{9}$

At our present state of knowledge and available technology 